Arthroskopie 2006 $\cdot 19: 67-74$ DOI 10.1007/s00142-005-0330-2

Online publiziert: 19. Januar 2006

(c) Springer Medizin Verlag 2006

H. Sadri ${ }^{1}$. J. Menetrey ${ }^{1} \cdot$ E. Kraus ${ }^{2} \cdot$ P. Hoffmeyer ${ }^{1}$

${ }^{1}$ Unité d'Orthopédie et Traumatologie du Sport, Clinique d'Orthopédie des Hôpitaux Universitaires de Genève . ${ }^{2}$ Département de Chirurgie Orthopédique,

Hôpital Sud Fribourgeois Riaz/Schweiz

\title{
Arthroskopische Behandlung des femoroazetabulären Impingements
}

konnte gezeigt werden, dass das FAI einen progredienten degenerativen Prozess auslösen und zur frühzeitigen Arthrose des Hüftgelenks führen kann [1, 9, 10, 11]. Das sich wiederholende Anschlagen des femoralen Kopf-Hals-Übergangs am Azetabulumrand resultiert aus einem verringerten Bewegungsumfang. Die Hüftgelenkbeweglichkeit hängt von der femoralen Kopfzu-Hals-Ratio und der Exkursionskurve des femoralen Halses, bevor Kontakt zum Azetabulumrand auftritt, ab $[15,21]$. Dies ist ähnlich der Mechanik beim totalendoprothetischen Hüftgelenkersatz. Deshalb können eine Verbreiterung des Femurhalses oder ein verringerter Offset zwischen Kopf und Hals als auch die lokale oder generalisierte Über-Überdachung des Azetabulums das Gelenkspiel verringern und das FAI potenzieren $[1,10,13]$. Ein verringertes Gelenkspiel ist ein bekannter Faktor, der bei Hüftendoprothesen zu repetitivem Kontakt zwischen Prothesenhals und dem Rand der azetabulären Komponente führt.

Die Erkenntnis der zerstörerischen Auswirkungen des FAI war die Veranlassung zur Entwicklung einer neuen, gelenkerhaltenden chirurgischen Methode [6, 7]. Deren Ziel ist eine zeitgemäße Behandlung, die den Degenerationsprozess, der durch das Impingement in Gang gesetzt wird, verlangsamt. In dieser Arbeit sollen die Hintergründe für eine solche Behandlungsstrategie dargestellt und eine neue arthroskopische Methode zur Behandlung dieser Pathologie präsentiert werden.

\section{Formen des femoroazetabulären Impingements (FAI)}

Basierend auf den Mustern und variierenden Stadien der chondralen und labralen Läsionen, die während der chirurgischen Exploration in situ beobachtet werden können, werden 2 verschiedene Typen unterschieden.

\section{Cam-FAI}

Das „Nockenwellen“- (Cam-)FAI wird durch ein Anschlagen eines abnormal geformten Femurkopfs mit erhöhtem Radius an das Azetabulum während kraftvoller Bewegung verursacht, insbesondere bei Flexion $[3,14]$. Die resultierenden Scherkräfte führen zu einer „Outside-inAbrasio“ des azetabulären Knorpels und/ oder zu seiner Ablösung vom Labrum und dem subchondralen Knochen in einem ziemlich konstant anterosuperior gelegenen Areal. Die Knorpelablösung führt im Gegenzug zu einer Ruptur oder einem Abtrennen des in erster Linie nicht beteiligten Labrums.

\section{Pincer-FAI}

Das „Beißzangen“- (Pincer-)FAI ist das Ergebnis eines linearen Kontakts zwischen Aztabulumrand und dem KopfHals-Übergang. Der Femurkopf kann eine normale Morphologie haben, und der Konflikt ist das Ergebnis einer azetabulären Abnormität (• Abb. 6b), häufig einer tion des Schädigungsmusters und dem dynamischen Nachweis seiner Ursache 
generalisierten oder lokal anterior zu weiten Überdachung (azetabuläre Retroversion; [10]). Die 1. Struktur, die in dieser Situation versagt, ist das azetabuläre Labrum. Wiederholte Kompression resultiert in einer Degeneration des Labrums mit intralabraler Ganglionbildung oder Ossifikation der Labrumbasis (• Abb. 6b), was zu einer zusätzlichen Vertiefung des Azetabulums und Zunahme der Über-Überdachung führt. Das andauernde Anschlagen des Kopfes, häufig im anterioren Bereich und mit chronischer Hebelwirkung des Kopfes auf das Azetabulum, führt zu einer Knorpelläsion in der „Contre-coupRegion“ des posteroinferioren Azetabulums. Das Pincer-FAI wird häufiger bei Frauen im Alter zwischen 30 und 40 Jahren gesehen, die einen Sport mit eine entsprechende Hüftbeweglichkeit betreiben. Das Cam-FAI hingegen tritt in der Regel bei jüngeren, athletischen Männern auf [ 1 , $7,10]$.

\section{Beschwerdesymptomatik}

Das FAI ist die schmerzhafte Manifestation einer frühzeitigen degenerativen Hüftgelenkerkrankung. Beginnende Arthrose, entweder die sogenannte primäre oder sekundäre Form (Perthes, Dysplasie, Epiphysenfugenlösung, posttraumatisch etc.) zeigen typischerweise eine ähnliche Symptomatik.

Intermittierende Leistenschmerzen, zumeist leicht medial oder lateral der Spina iliaca anterior superior, lassen an ein anterosuperiores Impingement denken. Langanhaltende sitzende Position wie beim Autofahren, Treppensteigen oder das Überkreuzen des Beins der symptomatischen Seite über das andere Bein, werden oft als schmerzhaft angegeben. Das superiore Impingement ist ebenfalls häufig, wobei in diesem Fall die Patienten über lateralseitige Hüftbeschwerden klagen. Das posteriore Impingement findet sich weniger häufig. Diese Patienten geben nächtliche Gesäßschmerzen an. Extension und Außenrotation in Rückenlage lösen das Impingementphänomen aus. Auf Nachfrage geben manche Patienten an, dass sie mit Kissen unter den Knien schlafen, der sogenannten „comfort position“. Bei Verdacht sind typische körperliche Untersuchungsbefunde vorhanden und eine weiterführende
Bildgebung wie Standardröntgen und Magnetresonanzarthrographie hilfreich.

\section{Körperlicher Untersuchungsbefund}

Die körperliche Untersuchung sollte eine Ganganalyse, Bewegungsprüfung, lokale Schmerzdiagnostik und spezifische Tests beinhalten. Eine verringerte Beweglichkeit gibt normalerweise Hinweis auf ein intraartikuläres Problem, dessen häufigste Ursachen die Hüftgelenkarthrose und das Impingementsyndrom sind.

Es gibt einige einfache, aber spezifische klinische Tests, die dazu dienen, die zugrunde liegende subtile Hüftgelenkerkrankung zu untersuchen. Der Impingementtest wird dazu verwendet, das Azetabulumrandsyndrom und anterosuperiore Impingement auszulösen. Die Hüfte wird in $90^{\circ}$ Beugung nach innen rotiert und adduziert. Diese Kombination von Bewegungen bringt den anterioren Anteil des proximalen Femurhalses in Kontakt mit dem vorderen Azetabulumrand, der im Allgemeinen die Lokalisation der Labrumschädigung darstellt. Dies ruft akute Schmerzen hervor, ausgehend von einem mobilen Os acetabuli oder einem rupturierten, degenerierten oder ossifizierten Labrum $[7,20]$. Eine weniger häufige Ursache für einen positiven Impingementtest stellt die azetabuläre Retroversion dar [16]. Beide anatomische Varianten können frühzeitig ein FAI bei Innenrotation verursachen. Es wurde berichtet, dass Symptome einer posterioren Labrumruptur durch Extension, Abduktion und Außenrotation ausgelöst werden können, die als Schmerzen und möglicherweise durch ein Klicken imponieren [4]. Jedoch sind posteriore Labrumrisse selten, und viel häufiger leiden diese Patienten an Ossifikationen des posteroinferioren Labrums, sekundär durch eine idiopathische ossäre Metaplasie des Labrums (• Abb. 6b).

\section{Radiologische Diagnostik}

Zur Beurteilung des FAI werden eine a.p. Übersichtsaufnahme des Becken im Stehen sowie eine "Cross-table-Aufnahme“ in neutraler Hüftrotation angefertigt. Die Röntgenbilder erscheinen zunächst normal. Bei genauerer Betrachtung fallen je- doch Abnormitäten auf wie eine knöcherne Prominenz am anterolateralen KopfHals-Übergang, die am besten auf der lateralen Aufnahme gesehen wird, eine Aspherizität des Femurkopfs, z. B. als sog. „Pistolgrip-Deformität“ [7] und Veränderungen am Azetabulumrand wie ein Os acetabuli oder eine gedoppelte Linie, die sich bei einer Randossifikation zeigt. Im Bereich des Oberschenkelhalses zeigen sich mitunter fibrozystische Veränderungen („Impingementpits"), die Indikator eines Impingements sind [7]. Morphologische Veränderungen, die das Azetabulum und/oder das proximale Femur betreffen, wie Retroversion, relative anteriore Über-Überdachung, Coxa profunda, Protrusio acetabuli, Coxa vara, extreme Coxa valga können durch systematische Beurteilung der Nativröntgenaufnahmen aufgedeckt werden.

Die Stellung des Azetabulums [13, 16] kann ebenfalls auf Nativröntgenbildern vermessen werden. Eine a.p. Aufnahme des Beckens ist bei dieser Indikation optimal, wenn die Spitze des Steißbeins mit einem Abstand von 1-2 cm senkrecht genau über der Symphyse liegt [7, 19]. Danach werden Linien vom anterolateralen Erker entlang der anterioren und posterioren Projektion des Pfannenrands gezogen.

Wenn die posteriore Linie lateraler als die anteriore zu liegen kommt, ist das Azetabulum antevertiert. Wenn dagegen die Begrenzung der anterioren Wand lateral der hinteren zu liegen kommt, ist das Azetabulum retrovertiert. Eine Achterfigur erscheint, wenn die Vorderwand die Hinterwand auf einer der Skizzen kreuzt. Dieses wird „cross over sign“ genannt [16] und entspricht einem prominenten anterioren Rand, der eine relative Retroversion des proximalen Azetabulums verursacht. Wenn die nachgezogenen Begrenzungen auf gleicher Höhe liegen, spricht man von einer Neutralstellung des Azetabulums.

Bei Patienten mit Hüftschmerzen, deren Röntgenbilder unauffällig sind, und bei Patienten mit auffälligem Befund, aber schwer zu beurteilenden Abnormitäten, ist die MRT das bildgebende Verfahren der Wahl. Es empfiehlt sich, routinemäßig radiär geschichtete MR-Arthrographien anzufordern, die anhand eines gut etablierten Protokolls angefertigt werden [12], um das Labrum und den azetabulären Knorpel beurteilen zu können. MR- 
Arthrographien sind auch in der Lage, eine Aspherizität des Femurkopfs, fibrozystische Veränderungen („Impingementpits") oder eine Ossifikation des Pfannenrandes nachzuweisen oder zu bestätigen, die ihrerseits die Folge des Impingements sind. Der Mangel an Spherizität am KopfHals-Übergang (d. h. Konvexität anstatt von Konkavität) wird fehlender Offset am Kopf-Hals-Übergang genannt. Dieser konvexe Anteil des Kopf-Hals-Übergangs, der auf den radiären Sequenzen gesehen werden kann, ist typischerweise für das Impingement verantwortlich und sollte chirurgisch abgetragen werden. Anhand der MRT können diese Pathologie nachgewiesen und die Quantität des zu entfernenden Knochenanteils bestimmt werden.

MR-Arthrographien sind sehr sensitiv und spezifisch, um labrale und chondrale Läsionen nachzuweisen, aber haben ihre Grenzen bei der Diagnostik adhärenter subchondraler Knorpelablösungen (blasige Aufwerfung des Knorpels). Jedoch werden auch chondrale Lappen bei dieser Untersuchung im Vergleich zur Arthroskopie häufig übersehen.

\section{Therapie}

Bei persistierenden Schmerzen oder Therapieresistenz bei konservativer Behandlung (nach 3-6 Monaten) wird eine frühzeitige chirurgische Therapie nach Diagnosesicherung empfohlen, um das Hüftgelenk vor weiterer Zerstörung zu schützen. Die operative Behandlung des FAI konzentriert sich auf die Verbesserung der Hüftbeweglichkeit und Verminderung des femoralen Anschlags gegen den azetabulären Rand $[1,7,10,20]$.

\section{- Die angewendete Behandlungsmetho-} de hängt von verschiedenen Faktoren ab, aber wird hauptsächlich von der zugrunde liegenden morphologischen Fehlform bestimmt, die das Impingement verursacht.

In den meisten Fällen koexistiert eine Kombination anatomischer Aberrationen oder kausaler Faktoren. Ist die Lokalisation des Impingements identifiziert, kann eine effektive operative Therapie mit guten mittelfristigen Ergebnissen erfolgen [1]. Die Hüftarthroskopie ist eine sehr attraktive

Arthroskopie $2006 \cdot 19: 67-74$

DOI 10.1007/s00142-005-0330-2

C) Springer Medizin Verlag 2006

H. Sadri · J. Menetrey · E. Kraus · P. Hoffmeyer

\section{Arthroskopische Behandlung des femoroazetabulären Impingements}

\section{Zusammenfassung}

Eine erst kürzlich etablierte, aber häufige Ätiologie der Hüftschmerzen beim jungen Erwachsenen ist das femoroazetabuläre Impingement (FAl), das eine leichte Form der frühzeitigen degenerativen Erkrankung des Hüftgelenks darstellt. Die klinischen Aspekte und diagnostischen Möglichkeiten sollen im Folgenden diskutiert und eine komplett arthroskopische Korrektur dieser Pathologie präsentiert werden. Mit dieser Technik können die azetabuläre Komponente ebenso wie die pathologischen Veränderungen am Femurkopf behandelt werden. Das schließt - falls notwendig - auch eine Labrumnaht mit ein. Neurologische Komplikationen durch lange Distraktionszeiten können durch die Anwendung einer invasiven Distraktionsmethode vermieden werden. Die Ergebnisse der arthroskopischen Behandlung sind mit denen der offenen chirurgischen Technik vergleichbar.

\section{Schlüsselwörter}

Femoroazetabuläres Impingement . Degenerative Hüfterkrankung · Hüftarthroskopie - Labrumnaht . Hüftdistraktion

\section{Arthroscopic treatment of femoroacetabular impingement}

\section{Abstract}

A newly established frequent etiology of hip pain in the young adult is femoroacetabular impingement, which is a subtle form of early degenerative hip disease. This contribution discusses clinical aspects and diagnostic features. A thorough arthroscopic correction of this pathology is presented. The technique treats the acetabular component as well as the head component. This also includes labral repair when necessary. Neurological complications are avoided during long distraction periods using invasive distraction. The results thus obtained are very similar to the open surgical technique.

\section{Keywords}

Femoroacetabular impingement . Degenerative hip disease · Hip arthroscopy . Labral repair $\cdot$ Hip distraction 


\section{Leitthema}

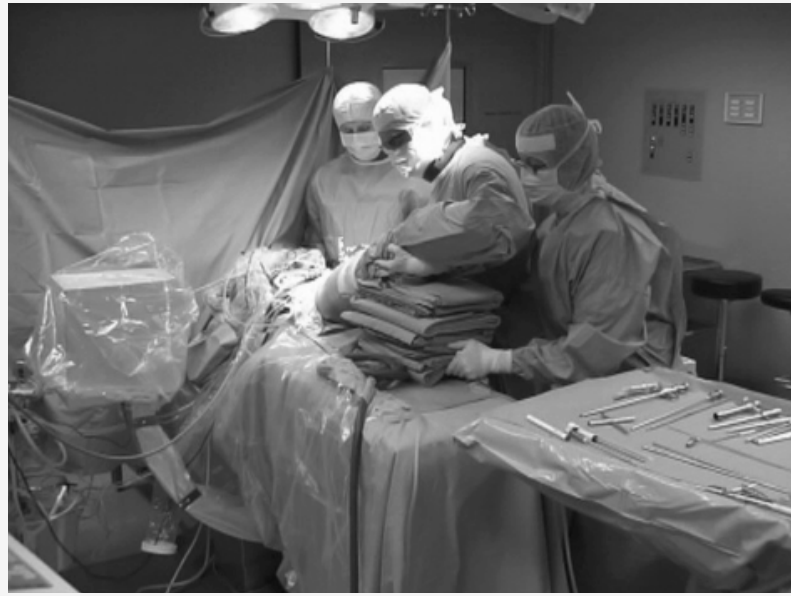

Abb. $1 \Delta$ Vorderansicht der linken Hüfte in $30^{\circ}$ Abduktion.

Gesamtübersicht mit Bildverstärker in der horizontalen Position.

Der Operateur steht hinter dem Patienten

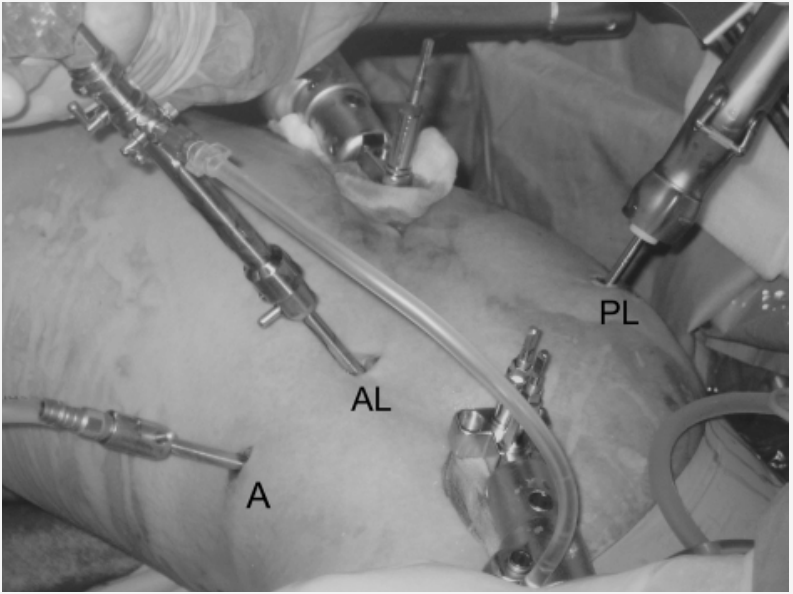

Abb. $2 \triangle$ Rechte Hüfte. Invasive Hüftdistraktion (Ansicht von kranial) mit posterolateralem $(P L)$, anterolateralem $(A L)$ und anteriorem $(A)$ Portal

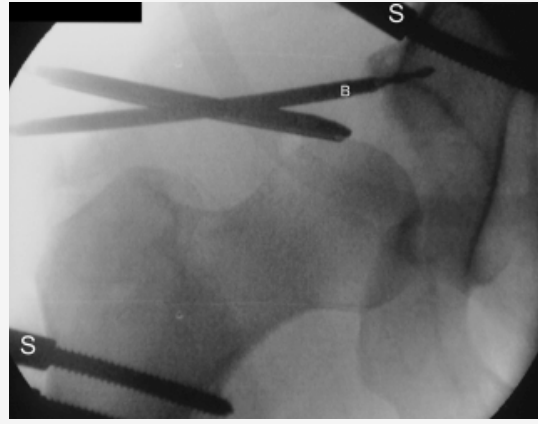

Abb. $3 \Delta$ Invasive Hüftdistraktion mit Bohrer (B) im ventrolateralen Pfannenrand zur Ankeranlage. Die Schanz-Schrauben (S) liegen auf Höhe des Trochanter minor und 1-2 cm proximal des Gelenkspalts
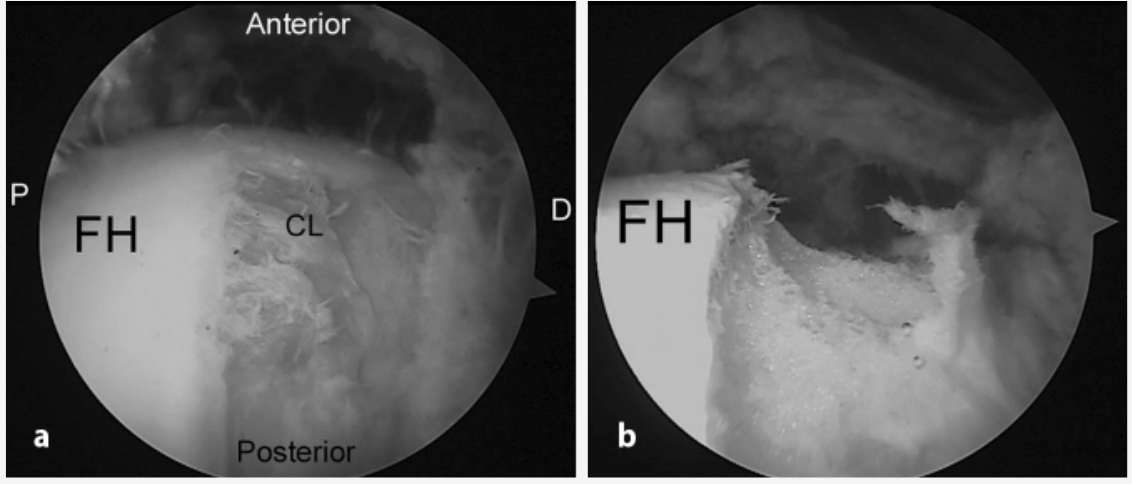

Abb. 4a, b $\Delta$ Rechte Hüfte, arthroskopische Blick ins periphere Kompartiment über das posterolaterale Portal: Knorpelläsionen am Kopf-Hals-Übergang des Femurs (Grad II-IV nach Outerbridge) vor (a) und nach (b) Korrektur des „, offsets". Femurkopfknorpel (FH), Knorpelläsion $(C L)$, proximal $(P)$, distal $(D)$, ventral (anterior), dorsal (posterior)

Rückenlage getestet. Die Autoren bevorzugen die Seitenlage, wobei die Lagerung (- Abb. 1) mit wenigen Unterschieden gleich wie für den klassischen seitlichen Zugang ist. Es werden 3 Standardportale verwendet (• Abb. 2), das

- posterolaterale,

- anterolaterale und

- anteriore.

Obwohl die Standardempfehlungen für Hüftarthroskopien in der Literatur zur Vermeidung neurologischer Komplikationen befolgt wurden (z. B. Verwendung einer sehr großen perinealen Abstützung), fanden sich bei den ersten 20 Fällen 2 mit einer langsam regressiven Pudendusläsion. Diese wurden wahrscheinlich durch eine länger als 90 min dauernde Trakti- on verursacht, die zur Korrektur der azetabulären Fehlform notwendig war. Auch mit zunehmender Erfahrung bzgl. der arthroskopischen Behandlung des FAI (mittlerweile über 200 Fälle) haben sich weder Operations- noch Traktionszeit verringert. Das hängt damit zusammen, dass immer mehr komplexe Fälle arthroskopisch operiert werden und in etwa $1 / 3$ der Fälle temporäre Labrumablösungen mit anschließender Refixierung notwendig sind. Im Falle einer Labrumrefixierung sind im Durchschnitt 4 Anker notwendig, wobei in einigen Fällen bis zu 8 Anker zur Anwendung kommen.

Aus diesem Grund wird vom Autorenteam seit dem Jahr 2000 eine invasive Distraktionsmethode eingesetzt (• Abb. 2), die eine isolierte Distraktion des Hüftgelenks erlaubt. Eine gleichzeitige Distrak- 
tion von Knie- und Sprunggelenk wird vermieden, sodass das Risiko eines Dehnungsschaden des N. ischiadicus gemindert wird. Die Vorrichtung vermeidet auch die peroneale Abstützung, die für die Schädigung des N. pudendus verantwortlich gemacht wird. Es trat bisher keine einzige Hauptnervenläson im Rahmen dieser Traktionsmethode auf. Bei über 200 Fällen hatten nur 2 eine leichte Parästhesie in der Region des N. cutaneus lateralis aufgrund einer Irritation durch das anteriore Portal und der postoperativen Schwellung. In beiden Fällen bildeten sich die Parästhesien spontan nach $48 \mathrm{~h}$ zurück. Die Rate der in der Literatur angegebenen neurologischen Komplikationen bei Verwendung eines Extensionstisches unter 120 min betragen zwischen 0,5 und $10 \%[5,8,17]$.

\section{- Die Verwendung eines Extensions- tisches wurde bei dieser Indikation aufgrund einer hohen Rate neurologi- scher Komplikationen aufgegeben.}

Seitlage und invasive Hüftdistraktion (DRMedical, Solothurn, Schweiz) werden aus folgenden Gründen bevorzugt:

- Kein Umlagern beim Übergang auf offene chirurgische Operation (wenn technische Probleme auftreten).

- In der Seitenlage fallen abgebrochene Instrumente und freie Körper in die Fossa und nicht nach dorsal. Eine arthroskopische Entfernung wird dadurch einfacher.

- Reduktion neurologischer Komplikationen durch eine lang anhaltende Distraktion bei schwierigen oder komplexen rekonstruktiven Hüftarthroskopien.

- Dynamischer Impingementtest nach Lösen des Fixateurs: Zum einen können nur so eine positives FAI verifiziert und eine freie Beweglichkeit der Hüfte nach der operativen Korrektur überprüft werden (maximale Flexion, maximale Innenrotation und schließlich maximale Abduktion). Das ist ohne Beeinträchtigung der Sterilität im Operationsfeld möglich. Obwohl die Bewegungsprüfung bei Verwendung eines Extensionstisches machbar wäre, könnte aufgrund der nichtsterilen Fußhalterung die Sterilität im Operationsfeld beeinträchtigt werden.
Die untere Extremität ist so abgedeckt, dass sie frei bewegt werden kann (• Abb. 1). Der Bildverstärker wird unter den Tisch durchgeschwungen, um so eine gute anteroposteriore Aufnahme zu ermöglichen (• Abb. 1). Der invasive Hüftdistrakteur wird in leichter Flexion und Distraktion an die Hüfte angebracht. Die Schanz-Schrauben werden $1 \mathrm{~cm}$ proximal zum Gelenkspalt und auf Höhe des Trochanter minor platziert (• Abb. 3 ). Die untere Extremität wird dann in $30-40^{\circ}$ Abduktion gelagert (• Abb. 1). Nach erfolgter Distraktion wird das Hüftgelenk mit einer langen Spinalnadel dekomprimiert und der Unterdruck dadurch aufgehoben. Unter Verwendung eines Kanülensystems wird das Arthroskop zunächst über das anterolaterale Portal eingebracht. Es empfiehlt sich ein $70^{\circ}$-Arthroskop, das anfangs eine bessere Sicht ermöglicht, besonders bei straffen Gelenken. Posterolaterales und anteriores Portal werden unter arthroskopischer Kontrolle angebracht. Das Hüftgelenk wird zunächst nach Luftinstillation, dann im flüssigen Milieu inspiziert. Die schließlich gewählte Behandlungsmethode hängt von der Art der vorhandenen Läsionen und vom Typ des Impingements ab. Die Therapieprinzipien werden in gleicher Weise wie bei der offenen chirurgischen Behandlung beibehalten $[1,10]$.

Das Gelenk wird mit dem Arthroskop in üblicher Weise beurteilt. Die Läsionen werden auf der azetabulären Seite identifiziert. Typische Befunde sind ausgefranste oder komplexe Risse des azetabulären Labrums im anterioren und superioren Quadranten. Beim Cam-Impingement sind gelenkseitige Risse des Labrums häufig mit juxtalabralen Knorpelschädigungen vergesellschaftet.

Nach dem diagnostischem "Rundgang“ folgt eine Kapsulotomie mit Hilfe einer Beaver-Klinge, typischerweise von 9-4 Uhr (bei einer rechten Hüfte). Dies ist unbedingt erforderlich, denn es ermöglicht dem Chirurgen, die Instrumente frei zu bewegen, ohne von der Gelenkkapsel behindert zu werden. Danach wird mit BeaverKlinge, Stanze und Shaver anterior und superior eine Kapsulektomie durchgeführt, um den vorderen und oberen Anteil des Kopfs darzustellen.

Das Arthroskop wird nun über das posterolaterale Portal eingeführt, die Instrumente über das anterolaterale. Der Kopf-

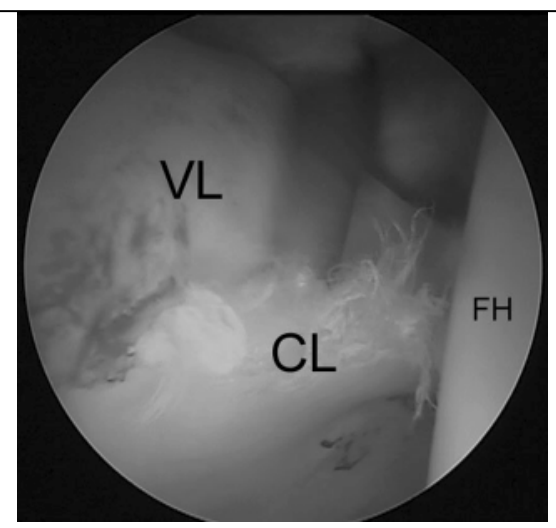

Abb. 5 \ Rechte Hüfte, arthroskopischer Blick ins zentrale Kompartiment über das posterolaterale Portal. Typische anterosuperiore Knorpelablösung am Übergang vom Labrum zum Knorpel bei Cam-FAI. Knorpellappen (CF), ventrales Labrum (VL) und Femurkopf $(F H)$

Hals-Übergang wird inspiziert, und die fettreiche Pars reflecta des Femurkopfs, die die versorgenden Gefäße enthält, dargestellt. Typischerweise finden sich in der Zone des Kopf-Hals-Übergangs Knorpelläsionen unterschiedlicher Grade (I-IV nach Outerbridge; - Abb. 4). Auf der azetabulären Seite werden evtl. vorliegende komplexe Labrumrupturen mit einem Shaver oder abgewinkelten radiothermischen Instrument (30 oder $45^{\circ}$ ) debridiert. Der Gelenkknorpel wird bis zu einem stabilen Bereich reseziert oder geglättet. Gelegentliche delaminierte Defekte und Knorpellappen müssen bis auf einen stabilen Restknorpel abgetragen werden (• Abb. 5).

Wenn das Labrum erhalten werden kann, normalerweise bei gelenkseitigen longitudinalen Partialrupturen (• Abb. 5), wird es vom Azetabulumrand abgelöst (- Abb.6a-c). Hierdurch wird wie bei der offenen Operationstechnik ein temporärer „Korbhenkelriss“ (• Abb. 6c) geschaffen $[1,10]$. Häufig werden dadurch labrale Ossifikationen sichtbar ( $\bullet$ Abb. 6b). Bei azetabulärer Retroversion oder labralen Ossifikationen werden diese mit einer Fräse abgetragen ( $\bullet$ Abb. $6 \mathbf{c}$ ). Selten wird auch ohne Vorliegen einer Pathologie der Azetabulumrand mit der Fräse angefrischt. In beiden Fällen wird das Labrum danach mit Hilfe kleiner resorbierbarer Anker mit reißfesten, nichtresorbierbaren Fäden (z. B. Biosuturetac, Arthrex, Karlsfeld, Deutschland) reinseriert ( $\bullet$ Abb. 7ad). Resorbierbare Anker sind zu bevorzugen, da sie bei zukünftigen Kontrollen die 


\section{Leitthema}
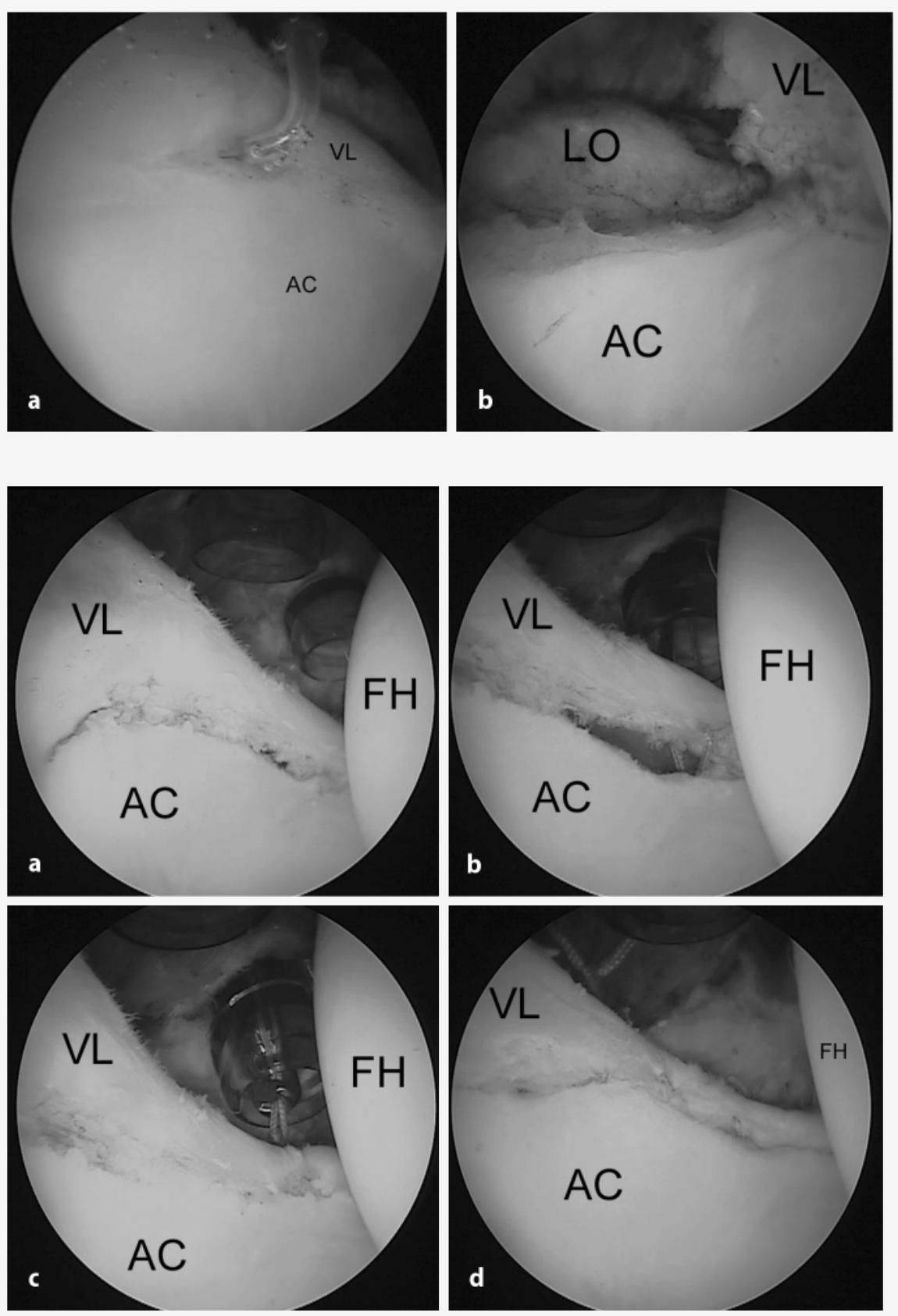

MR-Signale nicht beeinflussen. Ebenso stören sie nicht im Falle einer später notwendigen Implantation einer Hüftendoprothese. Zum Fadenshuttle wird reißfestes Fadenmaterial (z. B. Fiberwire, Arthrex, Karlsfeld, Deutschland) benötigt. Bei der 180 - bis $360^{\circ}$-Drehung reißen resorbierbare Fäden relativ häufig. „Suture passers“ und "Shuttletechnik“ sind erfahrungsgemäß die am wenigsten aggressive $\mathrm{Me}$ thoden der Labrumnaht (• Abb. 7a-d). In manchen Fällen erweist sich das Labrum in der Tat als sehr dünn, und Instrumente vom Typ „bird beak“ erscheinen zu massiv. Eine Labrumbreite von $5 \mathrm{~mm}$ erscheint daher als angemessenes Limit zur Reinsertion. Es ist notwendig, die Fäden auf der kapsulären Seite des Labrums zu knoten (• Abb. 7c), damit die Hüftgelenkmechanik nicht gestört und der Knorpel geschont wird.

Die Traktion wird anschließend aufgehoben und der Hüftkopf vom posterolateralen Portal aus inspiziert. Die fettreiche Pars reflecta am Kopf-Hals-Übergang wird nun dargestellt. Dies ist notwendig, da dieser Bereich die terminalen Äste der A. circumflexa medialis enthält und diese

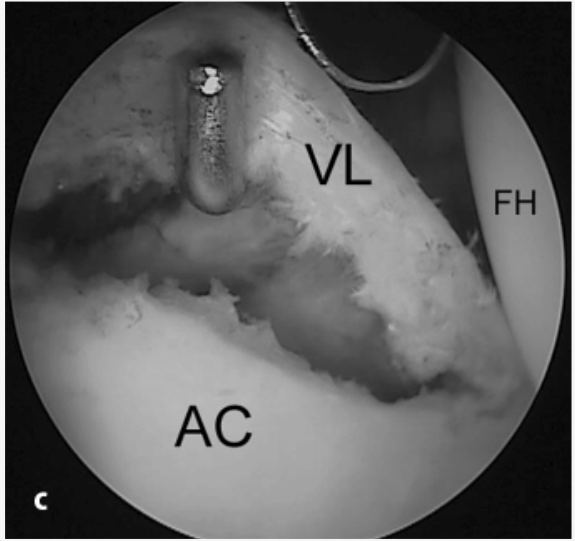

Abb. 6a-c $\Delta$ Ablösen des Labrum acetabulare zur Entfernung einer Labrumossifikation und Pfannenrandtrimmung: Ventrales Labrum vor Ablösung (a), Darstellung der häufig vorhandenen partiellen labralen Ossifikation (Crista, b), Transformation der Labrumruptur in eine Art Korbhenkelriss (c). Labrumossifikation (LO), ventrales Labrum (VL), Azetabulumknorpel ventral $(A C)$ und Femurkopf $(F H)$

Abb. 7a- $d<$ Labrumnaht mit bioresorbablen Ankern. Längsriss des ventrolateralen Labrums (a), Platzieren des 1. Ankers (b), arthroskopisches Knoten (c) und Ergebnis (d). Ventrales Labrum (VL), Azetabulumknorpel ventral $(A C)$ und Femurkopf $(F H)$

die wichtigste Arterie zur Blutversorgung des Femurkopfes ist [18]. Der nichtsphärische Anteil der Femurkopfs ist normalerweise durch einen Knorpelschaden gekennzeichnet (• Abb. 4a). Dieser Bereich wird reseziert, um eine Konkavität zu erreichen (-Abb. 4b).

Die radiären MR-Schichten helfen bei der Planung der Resektionstiefe. Intraoperativ kann der Bildverstärker zur besseren Quantifizierung der Resektion verwendet werden. Normalerweise ist eine Resektionstiefe von 7-10 mm erforderlich. Die abschließende dynamische Testung der Hüf- 
te ist jedoch der wichtigste Moment der Operation. Hier zeigt sich, ob die Resektion ausreichend ist. Wenn nicht, muss mit der Resektion fortgefahren werden, bis sich eine impingementfreie Beweglichkeit darstellen lässt.

Zur Resektion beginnen wir mit der Kamera im posterolateralen Portal und der Fräse im anterolateralen Portal. In dieser Stellung wird am lateralen Kopf-HalsÜbergang gefräst. Zum Fräsvorgang am anerioren Kopf-Hals-Bereich werden die Kamera in das anterolaterale und die Fräse in das anteriore Portal eingeführt. Die Abflusskanüle kommt nun im posterolateralen Portal zum Liegen. Der vordere Bereich des Kopf-Hals-Übergangs ist die Hauptregion des femoroazetabulären Impingements. Dieser Bereich führt typischerweise in Flexion und Innenrotation zur Impingementsymptomatik. Die Morphologie des Kopf-Hals-Übergangs wird während des Fräsvorgangs mit Hilfe des Bildwandlers kontrolliert. Die Resektion ist ausreichend, wenn bei mechanischer Testung in $90^{\circ}$ Beugung und maximaler Innenrotation eine impingementfreie $\mathrm{Be}$ weglichkeit erzielt ist.

\section{Nachbehandlung}

Postoperativ werden die Patienten an 2 Gehstützen für 6 Wochen in Teilbelastung von 5-10 kg mobilisiert. Nach 6 Wochen werden Kräftigungsübungen der Abduktormuskulatur begonnen. Schwimmen und Radfahren sind erlaubt, sobald dies vom Patienten toleriert wird. ImpactSportarten oder Wettkämpfe sind vor Ablauf von 6 Monaten nicht erlaubt.

\section{Ergebnisse}

In einer prospektiven Studie wurden arthroskopische Fälle mit chirurgischen Luxationen mit Trochanterosteotomie verglichen. Der Nachuntersuchungszeitraum betrug mindestens 2 Jahre. In dieser Studie galten folgende Einschlusskriterien:

- Leisten- oder Gesäßschmerzen über einen Zeitraum von mindestens 6 Monaten,

- Nichtansprechen auf nichtsteroidale Antiphlogistika und andere untergeordnete Schmerzmedikationen, 
- ein positives Impingementzeichen,

- ein pathologisches MR-Arthrogramm,

- keine Gelenkspaltverschmälerung auf der Beckenübersichtsaufnahme.

Alle Kriterien mussten vor dem Einschluss in die Studie erfüllt sein. Vorangegangene Hüftoperationen, Wirbelsäulenerkrankungen und Fälle, die eine Osteotomie von Femur oder Becken erforderlich machten, wurden ausgeschlossen.

Es waren 32 Fälle in der Arthroskopiegruppe mit einem mittleren Alter von 30 Jahren (19-54 Jahre). Die meisten Fälle benötigten eine kombinierte Korrektur des Azetabulums und Hüftkopfs (26 Fälle). Die mittlere Distraktionszeit betrug 175 min (120-240 min). Bei der Nachuntersuchung nach 2 Jahren fanden sich gleich gute Ergebnisse in beiden Gruppen, was die neue Methode bestätigt. Die WomacScores stiegen von präoperativ 57/100 (1596) auf 84/100 (50-99; $p<0,05)$.

\section{- Die Patientenzufriedenheit lag in der Arthroskopiegruppe beim Zweijahres- follow-up bei $82 \%$.}

Folgende Komplikationen traten auf: Zwei kleinere Hämatome und ein Fall einer Irritation der N. cutaneus lateralis, die sich nach 48 h spontan zurückbildete. Diese Ergebnisse sind mit denen von Beck et al. [1] vergleichbar, wenn keine Gelenkspaltverschmälerung auf den Röntgenbildern nachweisbar war. Die Autoren empfehlen eine relativ vorsichtige Nachbehandlung und hatten dementsprechend damit bei über 200 Fällen keine einzige Schenkelhalsfraktur zu beklagen. Obwohl die Zweijahresergebnisse für die offene und arthroskopische Behandlung ähnlich waren, favorisieren wir das arthroskopische Verfahren aufgrund der schnelleren postoperativen Rehabilitation. Während die Patienten nach der Arthroskopie bereits nach 68 Wochen voll belasten, dauert die Rehabilitation nach offener Behandlung 12 Wochen und länger. Der arthroskopische Eingriff kann zudem ambulant durchgeführt werden.

\section{Fazit für die Praxis}

Die arthroskopische Behandlung ist die Therapie der Wahl beim Vorliegen eines femoroazetabulären Impingements. Sie hat sich als gleichwertig effizient erwiesen wie die offene Technik, mit geringerer Morbidität nach einem 2-jährigen followup. Sie erfordert jedoch aufgrund ihrer Komplexität längere Distraktionszeiten an der Hüfte. Die invasive Distraktion der Hüfte hat die Gefahr für neurologische Folgeschäden aufgrund verlängerter Traktionszeiten vermindert. Obwohl die Ergebnisse im Zweijahres-follow-up sehr vielversprechend sind, sind jedoch noch längere Nachuntersuchungszeiträume notwendig, um die Überlebensrate der Hüftgelenke (bis zum endoprothetischen Ersatz) zu dokumentieren.

\section{Korrespondierender Autor Dr. H. Sadri}

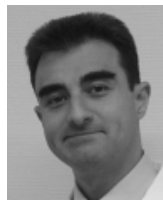

Unité d'Orthopédie et Traumatologie du Sport, Clinique d'Orthopédie des Hôpitaux Universitaires, 24, rue Michelidu-Crest CH-1211 Genf 14, Schweiz E-Mail: sadrih@hopfr.ch

Interessenkonflikt: Es besteht kein Interessenkonflikt. Der korrespondierende Autor versichert, dass keine Verbindungen mit einer Firma, deren Produkt in dem Artikel genannt ist, oder einer Firma, die ein Konkurrenzprodukt vertreibt, bestehen. Die Präsentation des Themas ist unabhängig und die Darstellung der Inhalte produktneutral.

\section{Literatur}

1. Beck M, Leunig M, Parvizi J et al. (2004) Anterior femoroacetabular impingement, part II. Midterm results of surgical treatment. Clin Orthop 418:67-73

2. Crawford JR, Villar RN (2005) Current concepts in the management of femoroacetabular impingement. J Bone Joint Surg [Br] 87(11):1459-1462

3. Eijer H, Myers SR, Ganz R (2001) Anterior femoroacetabular impingement after femoral neck fractures. J Orthop Trauma 15(7):475-481

4. Fitzgerald RH Jr (1995) Acetabular labrum tears. Diagnosis and treatment. Clin Orthop Relat Res 311:60-68

5. Funke EL, Munzinger U (1996) Complications in hip arthroscopy. Arthroscopy 12(2):156-159

6. Ganz R, Gill TJ, Gautier E et al. (2001) Surgical dislocation of the adult hip: a technique with full access to the femoral head and acetabulum without the risk of avascular necrosis. J Bone Joint Surg [Br] 83(8):1119-1124

7. Ganz R, Parvizi J, Beck M et al. (2003) Femoroacetabular impingement: a cause for osteoarthritis of the hip. Clin Orthop 417:112-120
8. Griffin DR, Villar RN (1999) Complications of arthroscopy of the hip. J Bone Joint Surg [Br] 81(4):604-606

9. Ito K, Minka MA 2nd, Leunig M et al. (2001) Femoroacetabular impingement and the cam-effect. $A$ MRI-based quantitative anatomical study of the femoral head-neck offset. J Bone Joint Surg [Br] 83(2):171-176

10. Lavigne M, Parvizi J, Beck M et al. (2004) Anterior femoroacetabular impingement, part I. Techniques of joint preserving surgery. Clin Orthop 418:61-66

11. Leunig M, Casillas MM, Hamlet M et al. (2000) Slipped capital femoral epiphysis: early mechanical damage to the acetabular cartilage by a prominent femoral metaphysis. Acta Orthop Scand 71(4):370375

12. Leunig M, Podeszwa D, Beck M et al. (2004) Magnetic resonance arthrography of labral disorders in hips with dysplasia and impingement. Clin Orthop 418:74-80

13. Myers SR, Eijer H, Ganz R (1999) Anterior femoroacetabular impingement after periacetabular osteotomy. Clin Orthop 363:93-99

14. Notzli HP, Wyss TF, Stoecklin CH et al. (2002) The contour of the femoral head-neck junction as a predictor for the risk of anterior impingement. J Bone Joint Surg [Br] 84(4):556-560

15. Rab GT (1999) The geometry of slipped capital femoral epiphysis: implications for movement, impingement, and corrective osteotomy. J Pediatr Orthop 19(4):419-424

16. Reynolds D, Lucas J, Klaue K (1999) Retroversion of the acetabulum. A cause of hip pain. J Bone Joint Surg [Br] 81(2):281-288

17. Sampson TG (2001) Complications of hip arthroscopy. Clin Sports Med 20(4):831-835

18. Sevitt S, Thompson RG (1965) The distribution and anastomoses of arteries supplying the head and neck of the femur. J Bone Joint Surg [Br] 47:560573

19. Siebenrock KA, Kalbermatten DF, Ganz R (2003) Effect of pelvic tilt on acetabular retroversion: a study of pelves from cadavers. Clin Orthop 407:241248

20. Siebenrock KA, Wahab KH, Werlen S et al. (2004) Abnormal extension of the femoral head epiphysis as a cause of cam impingement. Clin Orthop 418:54-60

21. Yamaguchi M, Akisue T, Bauer TW, Hashimoto Y (2000) The spatial location of impingement in total hip arthroplasty. J Arthroplasty 15(3):305-313 